\title{
A review of materials engineering in silicon-based optical fibres
}

\author{
Noel Healy ${ }^{1 *}$, Ursula Gibson ${ }^{2}$ and Anna C. Peacock ${ }^{3}$ \\ ${ }^{1}$ Emerging Technology and Materials Group, Newcastle University, NE1 7RU, UK \\ ${ }^{2}$ Physics Department, Norwegian University of Science and Technology, Trondheim \\ 7491, Norway \\ ${ }^{3}$ Optoelectronics Research Centre, University of Southampton, Southampton, SO17 \\ 1BJ, UK \\ E-mail: *noel.healy@ncl.ac.uk
}

\begin{abstract}
Semiconductor optical fibre technologies have grown rapidly in the last decade and there are now a range of production and post-processing techniques that allow for a vast degree of control over the core material's optoelectronic properties. These methodologies and the unique optical fibre geometry provide an exciting platform for materials engineering and fibres can now be produced with single crystal cores, low optical losses, tunable strain, and inscribable phase composition. This review discusses the state-of-the-art regarding the production of silicon optical fibres in amorphous and crystalline form and then looks at the post-processing techniques and the improved material quality and new functionality that they afford.
\end{abstract}

Keywords: Semiconductor materials, optical fibres, fibre fabrication, semiconductor photonics

\section{Introduction}

There is no doubt that the optical fibre and the silicon chip have both transformed the way in which humanity communicates and shares ideas. Over the last decade a number of research groups have been working to merge these society changing technologies to produce silicon-based optical fibres that can exploit the optoelectronic functionality of the semiconductor materials directly within the fibre geometry. Silicon based optical

fibres typically consist of a silica cladding and a silicon or silicon-alloy core material, and thus afford the optical fibre some of the functionality of the more traditional 'on-chip' silicon-on-insulator platform. This includes direct access to the electrical properties of the material and also the non-linear optical properties that can be four orders of magnitude greater than those of the standard silica optical fibre $[1,2]$. In a short span of time, there have been great strides made with regards the demonstration of devices that can harness the core material's properties for ultrafast optical signal modulation and 
broadband frequency generation [3-6]. These devices, being in fibre format, have the potential to be directly integrated with the existing optical fibre infrastructure, paving the way for all-fibre networks with much greater functionality.

Owing to its importance in the microelectronics industry, silicon has been extensively investigated to find ways of controlling its optoelectronic properties such as its electron mobility and energy bandgap. Investigations have included the introduction of dopants, strain and alloying materials, all of which have the common goal of enabling Moore's law, via continued CMOS scaling, beyond 2020 [7-10]. The silicon optical fibre has a very different geometry to CMOS based chips, which opens up new opportunities for engineering the material's properties. The large aspect ratio of the core, with high confinement in the radial direction facilitates extreme localisation of the engineering conditions. Furthermore, these conditions can be varied along the longitudinal axis of the fibre, greatly increasing the functionality. For example, it has been found that it is possible to use a laser to permanently modify silicon's bandgap energy by 'writing' anisotropic tensile stresses of up to $5 \mathrm{GPa}$ into the core material [11]. This approach enables the bandgap of the silicon to be varied along its length, which has the potential to greatly simplify the the fabrication and integration of optoelectronic components.

Though the semiconductor optical fibre platform has just undergone its first decade of active development, the potential of post-processing these fibres is a relatively new topic of research with some key advances already being demonstrated. This review article will highlight the significant advances that have been made to date, and explore the landscape for the application of these techniques to further enhance the functionality of the semiconductor optical fibre.

\section{Fabricating silicon optical fibres for post-processing}

In general, there are two complementary methods for the production of silicon optical fibres, the high pressure chemical vapour deposition (HPCVD) technique and the molten core drawing (MCD) process. In the context of producing fibres for postprocessing applications, both techniques offer advantages, for example the HPCVD technique can produce optical fibres with an amorphous core and the MCD process can produce fibres with highly crystalline cores; two very different starting points for post-processing $[12,13]$. In this section, we review both methods and pay particular attention to the material properties of the fibre cores.

\subsection{High pressure chemical vapour deposition}

It was in 2006 that the first silicon-core optical fibre was produced. Collaborators from Pennsylvania State University and the Optoelectronics Research Centre at the 


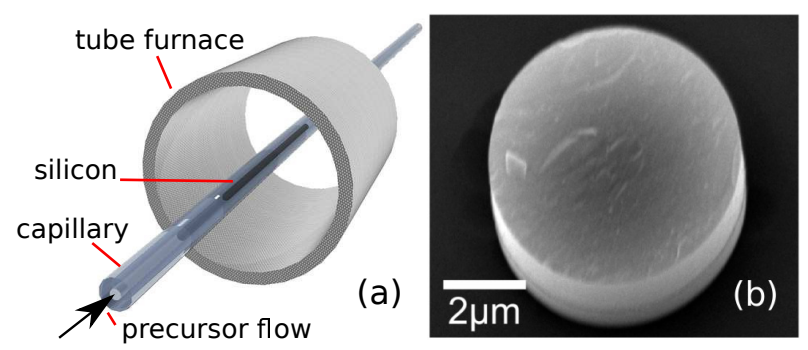

Figure 1. The HPCVD silicon fibre; a) A schematic of the deposition process. b) A small core silicon fibre with complete core filling.

University of Southampton used a modified HPCVD process to grow the silicon inside the pore of a silica capillary which acted as a low refractive index cladding and a template for the final structure [12]. In this instance, silica was chosen for the cladding material as it is compatible with standard optical fibres and is strong enough to withstand the high pressures required of the deposition process. The capillary is threaded through a small tube furnace and a mixture of silane and a carrier gas is forced through the capillary at a pressure of $35 \mathrm{MPa}$, a schematic of the process is shown in Fig. 1(a). The reaction is thermally initiated at the region of interest and, as the precursors decompose, the material is conformally deposited onto the near atomically smooth capillary walls (roughness values of $\sigma \sim 0.1 \mathrm{~nm}[14,15]$ ). To produce fibres that are amenable to postprocessing, it is important that the silicon completely fills the capillary pore as trapped air or gases can expand when heated and deform the entire structure. For silicon optical fibres, this is possible as the reaction's byproducts can diffuse through the capillary wall. Furthermore, careful control of the furnace's temperature profile can be used to deposit the core over lengths of several centimetres [16,17]; an image of a filled step index fibre can be seen in Fig. 1(b).

One of the major benefits that the HPCVD process has over other techniques is that is can be undertaken at much lower temperatures. Precursor decomposition requires significantly lower energy than that for melting bulk material and the silicon can be deposited at temperatures as low as $300^{\circ} \mathrm{C}[18]$. As a result, it is possible to deposit silicon in an amorphous, hydrogenated amorphous or poly-crystalline state, which provides a wide range of starting materials for post-processing applications. Fig. 2 shows the Raman spectra of various forms of silicon that can be deposited. Other advantages afforded by low temperature deposition include the mitigation of thermal expansion mismatching and the ability to make small core (sub-micron) fibres without the issues associated with elemental in-diffusion from the cladding into the core.

\subsection{Molten core drawing}

Molten core drawing was first demonstrated for making silicon optical fibre by the Ballato Group at Clemson University in 2008 [19] and has since been adopted by a number of groups worldwide [20-24]. The method is based on a modified fibre drawing 


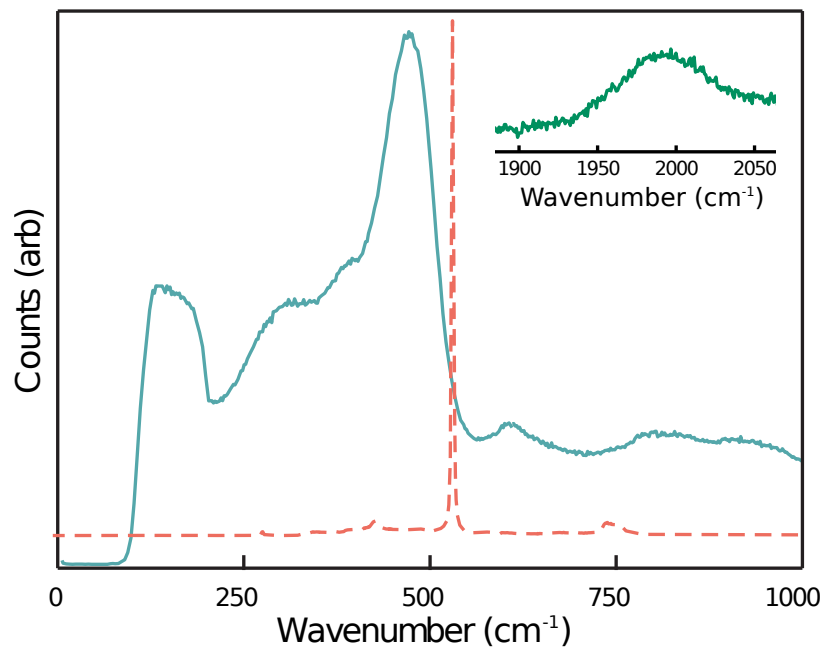

Figure 2. The Raman spectra of as-deposited silicon using the HPCVD process; blue line is amorphous, green line is the peak relating to the hydrogen-silicon bond of hydrogenated amorphous silicon, and dashed peach is poly-crystalline silicon.

process in which the preform is typically made from a silica tube that has been filled with silicon in rod or powder form. As schematically illustrated in Fig. 3(a), the preform is fed into the furnace where it is heated to above the core material's melting temperature before being drawn into a fibre. Though the silica cladding is above its glass transition temperature, it is still robust enough to act as a crucible for the molten silicon and the resulting fibres maintain their cylindrical symmetry when drawn, see Fig. 3(b). The key benefits of the MCD technique are that it can produce very long lengths of fibre (100's of kilometres) and that the cores consist of long millimetre-scale single crystal grains $[25,26]$.

With respect to the production of silicon fibres for post-processing, the high temperatures associated with the MCD technique mean that the core material is limited to being in a crystalline form. However, it does present the opportunity to do some reactive materials science in the drawing step and has been shown as a simple route to making optical fibres with silicon alloy cores, an example being the SiGe optical fibre [27]. The core size of fibres produced by the MCD process are relatively large with respect to those prepared by the HPCVD approach as the in-diffusion of oxygen from the cladding, can restrict the final achievable core size. This has been to some extent addressed by adding oxygen getters and diffusion barriers to the preform [21,28], which has enabled fibres to be drawn with core sizes as small as $4 \mu \mathrm{m}$. However, it should be noted that this is still quite large compared to the size required for single-mode operation at telecoms wavelengths $(350 \mathrm{~nm})$. Post-processing of the fibres can be an alternative route to enabling further core size reduction and fibres with nano-scale cores have been demonstrated [29]. 

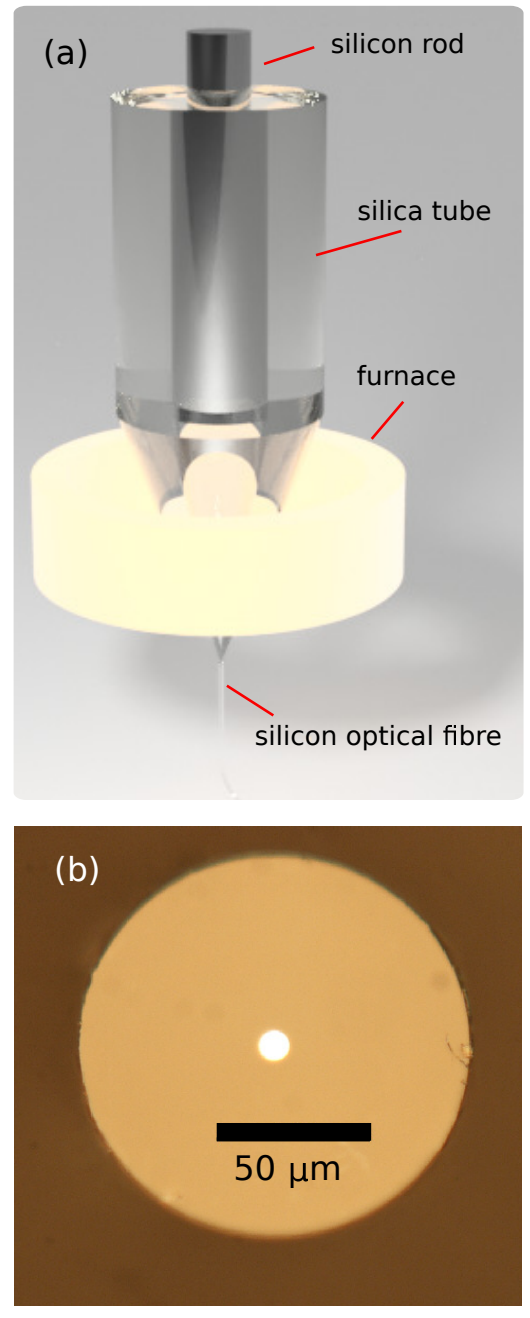

Figure 3. (a) A schematic of the MCD process and (b) a silicon-core optical fibre drawn by the MCD process.

\section{Post-processing techniques}

A key advantage of the silicon-core fibre platform is that it is possible to exploit the advanced post-processing techniques that have been developed for conventional glass fibres to optimize both the materials and the waveguide geometry. Important examples of this are heat treatments such as tapering and laser processing.

\subsection{Tapering via filament heating}

Tapering via filament heating is a well-established method used to post-process fibres with the primary function being to alter the waveguiding properties [30,31]. This process is typically conducted by heating and pulling the fibre from each end, which results in a re-scaling of the the core and cladding to smaller dimensions as it is stretched. However, an added benefit of this procedure for the silicon fibre is that the simultaneous heating and drawing of the molten semiconductor can help to improve the material quality as 


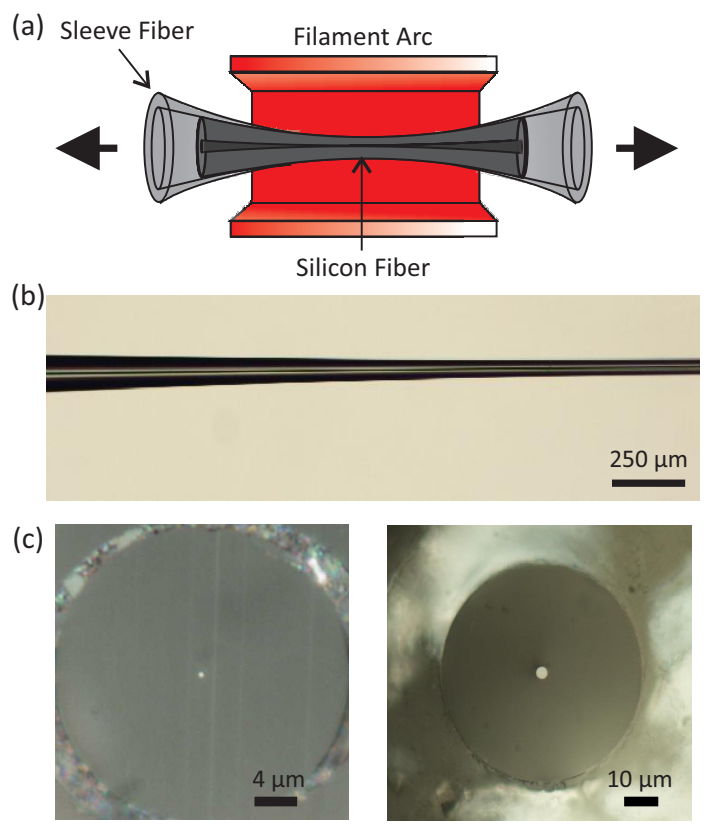

Figure 4. (a) Schematic of the tapering procedure in which the silicon fibre is sleeved into a longer capillary. The filament heats the combined structure, which is drawn in the direction of the arrows. (b) Longitudinal image of a tapered silicon-core fibre. (c) Microscope images of two polished fibre cross-sections, where the core diameters are $0.6 \mu \mathrm{m}$ and $4.8 \mu \mathrm{m}$, respectively.

the core crystallizes on cooling [32]. In order to maintain the integrity of the multimaterial fibres, a new tapering procedure has been developed in which the as-drawn silicon-core fibres are first sleeved inside a thicker capillary before heating and drawing, as illustrated in Fig. 4(a) [33]. As well as helping to strengthen the fibres as their dimensions are reduced, it also reduces the length of silicon fibre required for processing from several tens of centimetres to $\sim 1 \mathrm{~cm}$. The sleeved fibres can then be tapered using a standard glass tapering rig with appropriate choice of pulling speed and the filament power. During the processing, both the silica sleeve and fibre cladding soften, and fuse, while at the same time the silicon-core melts so that it flows through the reshaped cladding, before recrystallizing as it cools. The result is a smooth, continuous, tapering from the untapered fibre down to the waist, as shown in Fig. 4(b). This method is highly versatile and fibres have been fabricated with taper ratios of 1:2 to 1:20, as illustrated in Fig. 4(c). In all cases, the cladding diameters change proportionally to the core, and the total taper length is typically $\sim 2 \mathrm{~cm}$, of which there is a constant waist region of $\sim 1 \mathrm{~cm}$.

The material quality of the processed core materials have been assessed using both Raman spectroscopy and X-ray diffraction (XRD) measurements. Firstly, micro-Raman measurements were performed at the taper waist for the three fibres and compared with the as-drawn material, which in this instance was produced via the MCD process. The results for each of the tapered waists are shown in Fig. 5(a), together with a typical spectrum for the untapered fibre. A Voigt function (dashed curves) was used to 

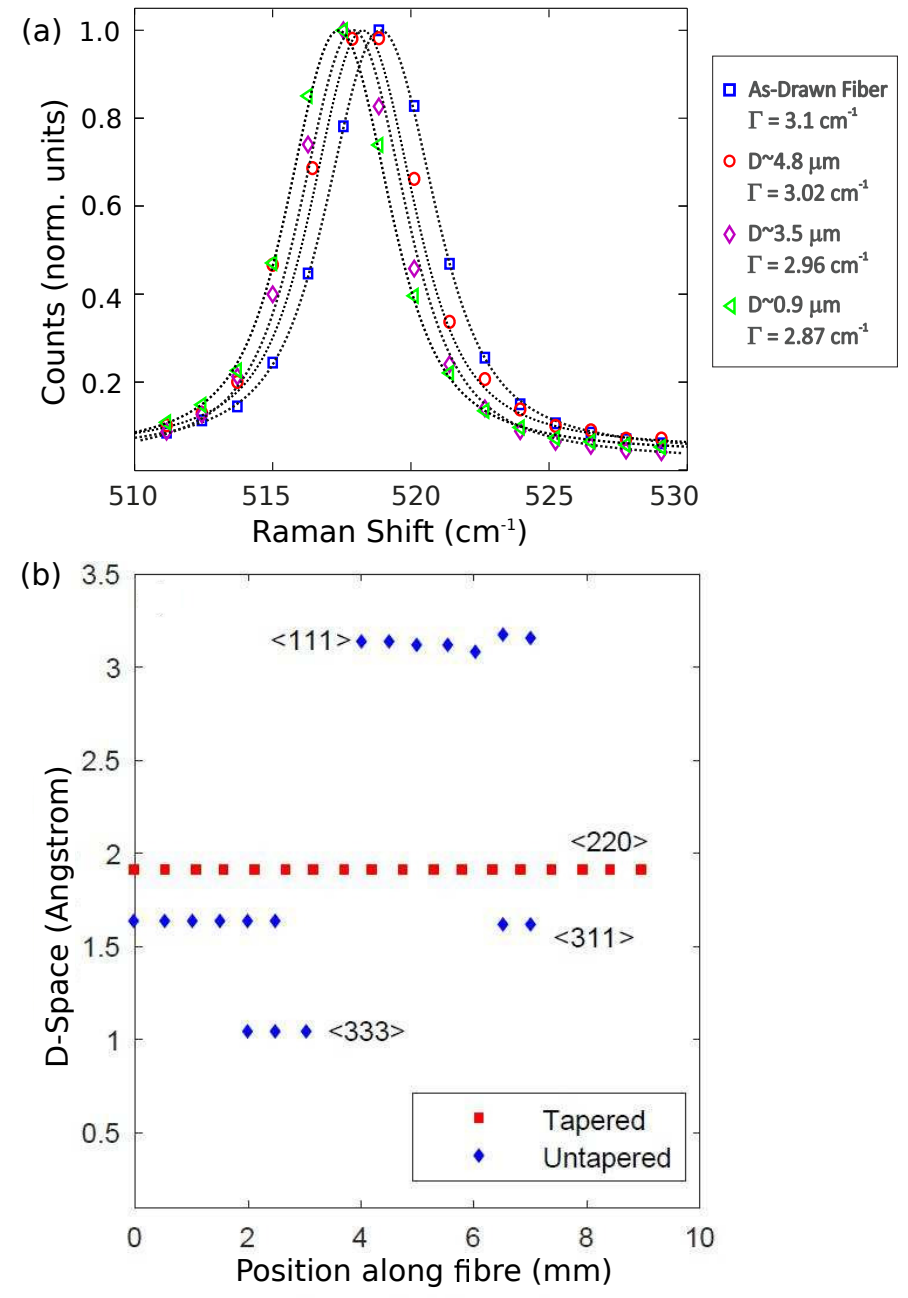

Figure 5. (a) Raman spectra for the silicon-core material taken for the as-drawn fibre and at the tapered waist sizes given in the legend. The dash curves are Voigt fits and the corresponding Lorentzian linewidths are given in the legend. (b) Lattice spacing of diffraction spots measured via XRD as a function of position along a section of the as-drawn fibre (blue) and tapered waist (red). Different d-spacings correspond to different crystallographic planes, as labelled.

fit the spectra to deconvolve the Lorentzian peak of the material from the instrument broadening, which is Gaussian shaped, with the extracted Lorentzian widths given in the legend. For comparison the silicon reference has a width of $\Gamma=2.7 \mathrm{~cm}^{-1}$. The estimated Raman widths of the measured fibres indicate that there has been an improvement of the poly-crystalline core material during the tapering process, in accordance with previous results for germanium core fibres [32]. Furthermore, the improvement appears to be more pronounced for the larger taper ratios. This trend is not unexpected as the smaller cores experience a higher cooling rate, which suppresses the number of nucleation sites [26,34]. The slight downshifting in the position of Raman peaks in the tapered fibres from the expected value of $520 \mathrm{~cm}^{-1}$ has been attributed to residual strain associated with differences in the thermal expansion of the core/cladding materials [17]. 
To investigate the crystallinity further, subsequent measurements were performed in a $D \sim 2 \mu \mathrm{m}$ tapered core fibre using a high-energy, micro-focused X-ray beam generated by a synchrotron light source [35]. By scanning the X-ray beam along the fibre length, it was possible to map the crystal grain size within the tapered region. The complete mapping of the crystallinity within two $\sim 1 \mathrm{~cm}$ long sections, corresponding to the asdrawn fibre (blue diamonds) and the tapered waist (red squares), is shown in Fig. 5(b). The as-drawn fibre is clearly made up of several millimetre long crystal grains, some of which overlap, in agreement with previous measurements of these fibres [21]. However, the tapered region is found to consist of only a single grain which extends over a length of $9 \mathrm{~mm}$, verifying that the crystallinity has been improved by the tapering process.

\subsection{Laser processing}

Owing to the versatility of laser processing, there are a number of research groups using such laser treatments to modify the optoelectronic and microstructural properties of semiconductor optical waveguides $[11,34,36-41]$. The fine control of the processing parameters, compared to rapid oven or photothermal [42] annealing, has led to significant improvements and control of their properties. Investigations have included the laser assisted crystallization/recrystallisation of the core material as well as the local modification of stress and material composition. Laser treatment is also being used to derive interesting encapsulated semiconductor structures within the glass cladding $[27,43,44]$.

While hot-filament treatment of the fibre indiscriminately heats both the core and cladding, lasers can be much more selective and localised when the appropriate wavelength is selected. Furthermore, pulsed laser treatments using above bandgap energy radiation can facilitate materials engineering at the core-cladding interface [39]. Highly localized heating and variable irradiation durations create conditions in which both kinetics and equilibrium thermodynamics are important, making these fibres a platform for the exploration of materials processing conditions not conventionally accessible. The two primary methods for laser recrystallization that have been explored are: 1) heating the core by direct optical absorption, and 2) heating the core by thermal conduction from a laser heated cladding. In the first case, the core is illuminated with photon energies above the energy bandgap of the core, but within the transparency range of the glass cladding. This allows for strong thermal isolation of the heated core material and the result can be used to strongly tune material properties such as the strain. In the latter case, the cladding is heated using infrared radiation that is absorbed by the cladding and energy is transferred to the core via thermal conduction so as to induce melting. In a recent paper, Gumennik et al used a diode laser combined with $\mathrm{CO}_{2}$ treatment to impart energy to the core and cladding simultaneously [43].

The general set-up for laser treatment involves a source of coherent radiation, a translation stage, and, in some cases, simultaneous monitoring of the emission from 


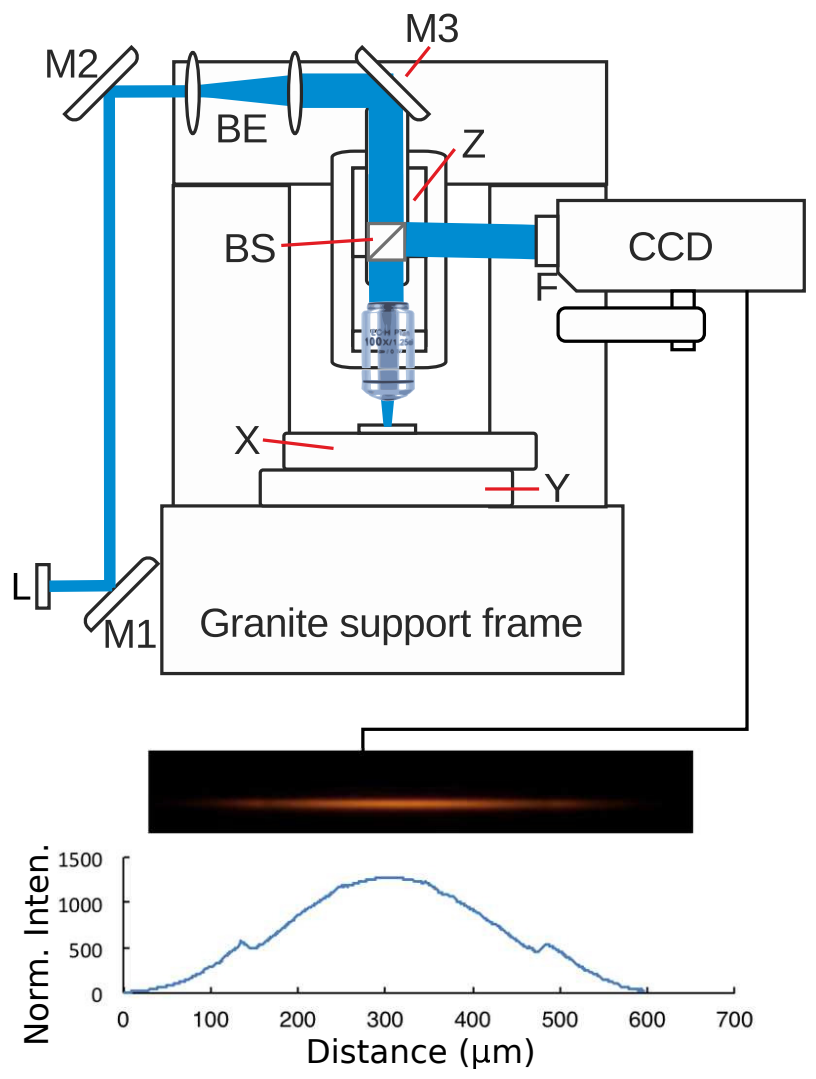

Figure 6. A schematic of a typical laser crystallization set-up. M1, M2, M3 are dichroic mirrors, $\mathrm{BE}$ is a beam expander, $\mathrm{BS}$ is a beam-splitter, $\mathrm{F}$ is a notch filter with a centre wavelength positioned at the laser's (L) wavelength, and $\mathrm{X}, \mathrm{Y}$, and $\mathrm{Z}$ are a set of programmable stages. The CCD can be used to capture the emission from the core.

the core, see Fig. 6. Imaging of the process is possible due to the higher visible light emissivity of the core materials (compared to the glass encapsulant) at the temperatures reached $[27,39]$.

\section{Materials engineering via post processing}

\subsection{Single crystal growth}

As discussed briefly in Section 3.1, the formation of large crystalline regions along the length of the core is desirable for optical applications as scattering losses are suppressed. Provided that the drawing conditions are optimised, the MCD technique can produce fibres that have large (millimetre-scale) crystals in the core [42, 45]. However, the core diameters are then restricted to 10's of micrometres, which can be too large for many optical applications [20]. Conversely, the HPCVD process can produce optical fibres with small cores, but also with small crystals along the cores length $(\sim 1 \mu \mathrm{m})[46,47]$. Though furnace-based thermal annealing has been used to reduce the losses of crystalline core fibres [48], laser processing and hot-filament tapering have emerged as powerful post- 
processing technologies for producing fibres with arbitrarily long single crystal cores so that the losses can be commensurate with the silicon photonics planar technology $[34,36]$.

As silicon is a unary system, solidification involves a competition between the nucleation of new grains versus epitaxial growth on existing grains. Nucleation of new crystallites is a time-dependent statistical phenomenon, so the probability of creating long single crystal regions in the fibre is increased by a rapidly moving solidification front. A large temperature gradient, as is afforded by laser and hot-filament heating, will suppress nucleation ahead of the main solidification front. If rapid scanning of a large axial temperature gradient region is performed, and the radial gradient of temperature is small, the likelihood of epitaxial growth is high. This was demonstrated by the authors of this review [34], where $10 \mu \mathrm{m}$ diameter silicon-cores were melted and re-solidified with a $\mathrm{CO}_{2}$ laser. The temperature gradients of $10^{4} \mathrm{~K} \mathrm{~cm}^{-1}$ permitted centimetre length single crystal fibre cores to be grown and the loses were reduced from $>20 \mathrm{~dB} \mathrm{~cm}^{-1}$ to $<2 \mathrm{~dB} \mathrm{~cm}^{-1}$, see Fig. 7. The growth of long single crystals has also been replicated with SiGe core fibres where the crystal lengths are seemingly only limited only by the travel of the translation stages [39].

An alternative approach to single crystal growth was undertaken in Ref. [11]. Using an argon ion laser with an emission wavelength of $488 \mathrm{~nm}$, the formation of $\sim 300 \mu \mathrm{m}$ length single crystals in cores of $1.7 \mu \mathrm{m}$ were observed. This technique was later extended by Ji et al. [36] to produce lengths of up to $1.6 \mathrm{~mm}$. The photon energy of the laser that was used by both teams is greater than the bandgap energy of silicon and is absorbed by the core, thus, presenting the opportunity for large departures from thermal equilibrium. Ji et al. suggest an optimal temperature gradient, based on simulations, of a few thousand $\mathrm{K} \mathrm{cm}^{-1}$ and discuss the advantages of keeping the cladding at a low temperature during remelting of the core. However, their simulations do suggest that, to fully melt the core, the cladding exceeds the softening point of the glass, at least in the interface region. In this instance the authors have reported optical losses of $<1 \mathrm{~dB} \mathrm{~cm}^{-1}[36]$.

The hot-filament tapering process outlined in Section 3.1 has also shown that large crystals of greater than $9 \mathrm{~mm}$ can be produced in the taper waist and, though the temperature gradients were not a focus of this study, in general, faster cooling rates have been associated with improved crystallinity. The material improvements obtained via hot-filament tapering also have corresponding loss reductions that are comparable to laser grown cores $\left(\sim 2 \mathrm{~dB} \mathrm{~cm}^{-1}\right)$ and both processes are complementary, which is an exciting prospect for the future of post-processed semiconductor optical fibres. 


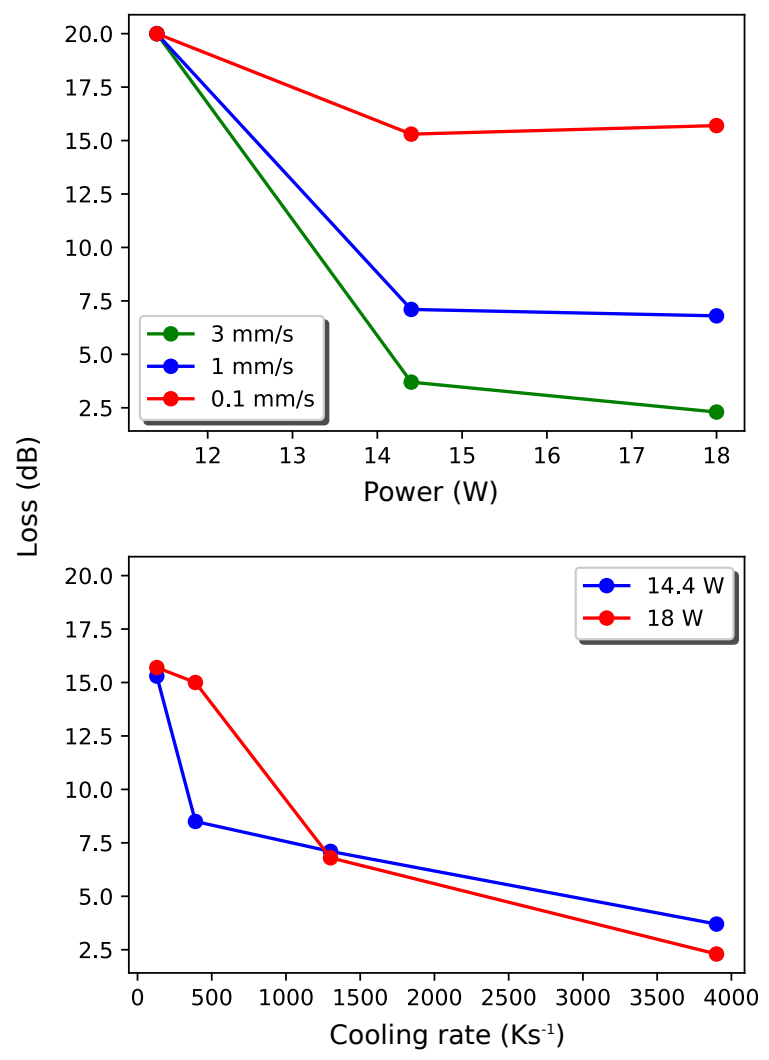

Figure 7. (a) Loss measurements for fibres that were recrystallised with a $\mathrm{CO}_{2}$ laser. (b) The same data as (a) but plotted as a function of temperature per second.

\subsection{Strain effects}

As the coefficients of thermal expansion of the core and cladding are different, the fabrication of crystalline silicon optical fibres produces an inevitable residual strain. By controlling the drawing conditions silicon fibres fabricated via the MCD process can have compressive or tensile strain across the core as there is a complex interplay between the softening of the cladding and the expansion of the core on solidification. Fibres fabricated in crystalline form using the HPCVD process (i.e. deposited at temperatures $>500^{\circ} \mathrm{C}$ ), display only a tensile strain on the core as the material reduces its volume when cooling to room temperature. If the fibre is first produced with an amorphous core, and subsequently crystallised in a furnace, a strong tensile strain of $\sim 1 \%$ can be induced. This is owing to silica being a native oxide of silicon, thus permitting a very strong bond to form at the core-cladding interface. When the core is crystallised it has a substantial decrease in volume; in this case the strain is somewhat reduced as the silica is heated above its glass transition temperature in the process. In all of these cases the strain is predominantly hydrostatic, which means that the optoelectronic properties are not significantly affected. Using a laser to selectively melt the core material provides 
a route to thermal, temporal and spatial control of this process, which allows one to develop more complex strain profiles in the fibre. A remarkable outcome of this is the production of fibres with tunable anisotropic strain that can be used to modify the core material's energy bandgap [11]. Fibres with amorphous cores of $1.7 \mu \mathrm{m}$ diameter were treated with a $1 \mathrm{~W}, 488 \mathrm{~nm}$ light source. Tensile strains up to $5 \%$, Fig. 8(a,b), were observed by Raman and XRD, and photoconductivity measurements were used to determine that bandgaps could be reduced to almost half that of bulk silicon, $0.59 \mathrm{eV}$, see Fig. 8(c). With such a shift, one can envision a silicon fibre that can guide telecoms light and have detectors written anywhere along its length.

$\mathrm{A} \mathrm{CO}_{2}$ laser can also be used to induce break-up of the silicon-core and produce a periodic linear array of spheres that can have either compressive or tensile values; the type of strain can be selected and tuned by varying the composition and temporal temperature profile $[27,43]$. Recent results on fibres made from sol-gel preforms [49] report somewhat lower levels of stress than are typically seen in silicon-core fibres, but it is likely this data was collected on the polished surface, and the proximity of the beam to the glass interface was not clear. Removal of the glass relieves the stress, so care must be taken when comparing data from etched or polished cores versus measurements through the glass cladding.

\subsection{Bragg gratings}

To date, two methods have been explored to make periodic modification in the axial properties of the semiconductor fibres, demonstrating the potential of these materials for use in Bragg grating structures [27]. The primary mechanisms are stress modification for femtosecond writing of a grating in silicon-core fibres, and compositional alterations for long period gratings in SiGe core gratings. In the former case, the grating was written in a fibre previously annealed to reduce losses. Bragg gratings for telecom wavelengths were written using short pulses $(517 \mathrm{~nm}, 220 \mathrm{fs}, 100 \mathrm{~nJ})$ which interact strongly with the silicon in the vicinity of the interface with the glass cladding, see Fig. 9. This introduced a periodic strain field in the core and cladding that was sufficient to give a narrowband reflected signal at the design wavelength. However, damage introduced into the glass during the process resulted in an overlaid stress field that was not perfectly periodic, leading to broadening of the reflected peak. Both birefringence in the cladding glass and variations of the Raman signal were used to assess the strain field [27].

Periodic compositional features were written in SiGe-core fibres by varying the velocity of the solidification front during laser processing [39]. Both on/off cycling of a $\mathrm{CO}_{2}$ laser, and explicit variation of the fibre velocity during solidification were used to inscribe periodic compositional changes in the core, with periods on the order of $100 \mu \mathrm{m}$, see Fig. 10. The largest reported variations in Ge concentration were approximately $3 \%$, corresponding to a large index change. No optical results were provided, but such gratings could potentially be of value for long wavelength applications. 

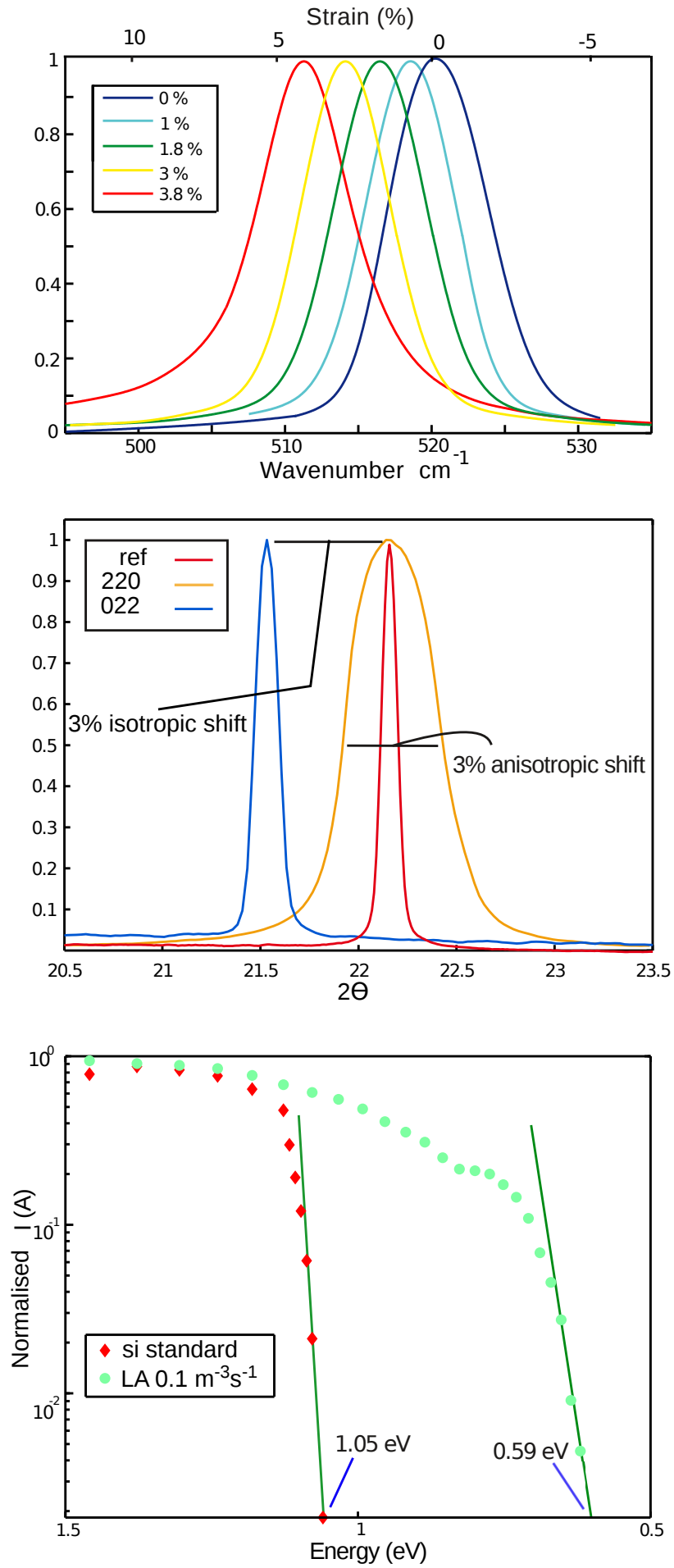

Figure 8. Laser processing of silicon fibre produced by the HPCVD technique. (a) Raman spectra demonstrating the tunabilty of the strain. (b) XRD peaks showing asymmetry of the strain. (c) Photoconduction measurements of a laser processed silicon optical fibre compared to a silicon standard.

\section{Perspectives and outlook}

The optical properties of the silicon-core fibres presented in this article clearly highlight their potential for use in a range of optoelectronic applications, as outlined below. 


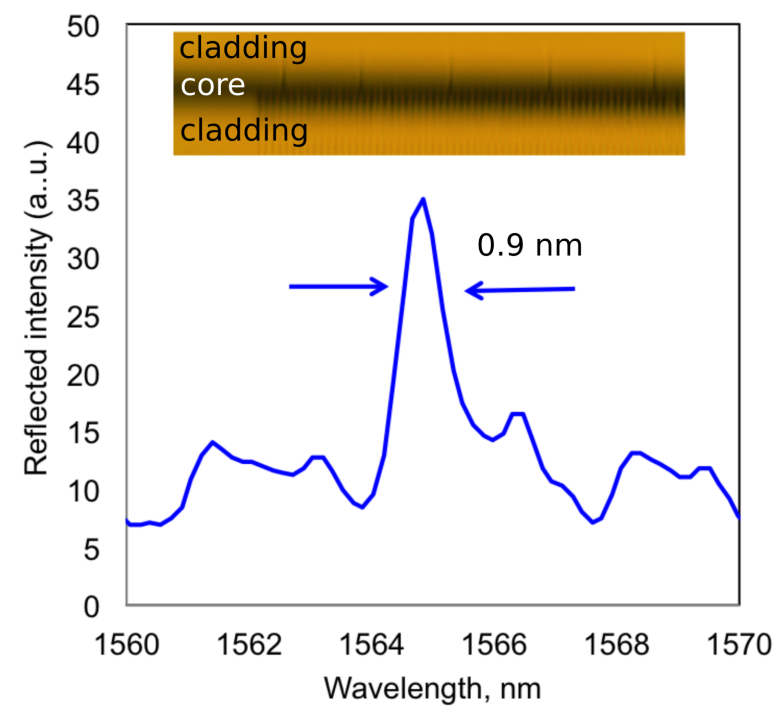

Figure 9. The reflected spectrum from a Bragg grating produced in a silicon optical fibre with a $517 \mathrm{~nm}$ femtosecond laser. Inset is on optical microscope image of the inscribed section of fibre.

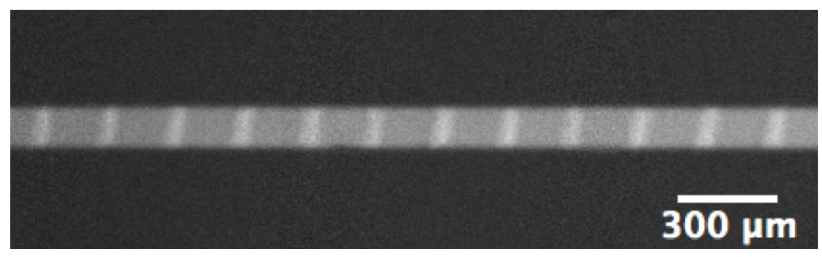

Figure 10. Periodic compositional engineering produced in a SiGe core optical fibre. In this instance a pulsed $\mathrm{CO}_{2}$ laser was used to produce Ge rich regions along the fibre's length

\subsection{Non-linear optics}

The combination of low losses and small core sizes, particularly in the tapered structures, are opening the door to the observation of a wide range of non-linear processes. Many of the early demonstrations focused on making use of the ultrafast speed of the Kerr non-linearity to realize devices such as optical modulators [5] and switches [50]. However, as control over the fabrication procedures has improved, more recently it has been possible to tune the dispersion properties of these fibres through the core size [51], making it possible to extend the non-linear measurements to the observation of broadband wavelength conversion via four-wave mixing [4]. In particular, such wavelength conversion is of interest for generating new sources of light in the infrared region where traditional glass fibres suffer from high losses. By further reducing the losses, we anticipate that these fibres could be used for the observation of a wide-array of non-linear dynamics extending from the telecoms band and well into the mid-infrared, where applications include surgery, imaging and sensing. Furthermore, there have been a number of theroetical papers that predict that the taper structures can be used for 


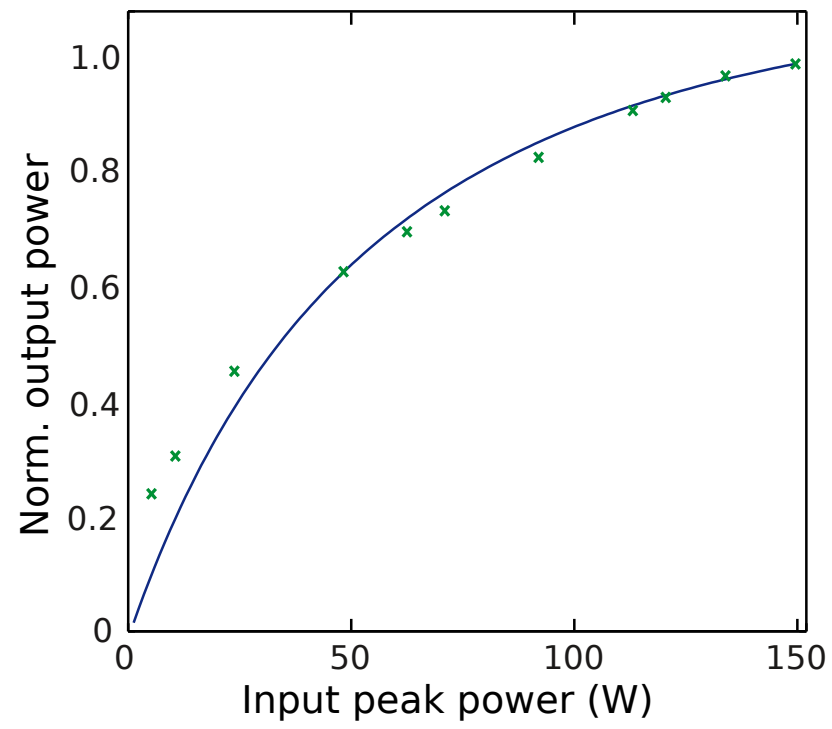

Figure 11. Non-linear absorption of a silicon optical fibre that has been tapered from a core diameter of $12 \mu \mathrm{m}$ to $2 \mu \mathrm{m}$.

pulse shaping and compression [52,53], which will be an exciting avenue of exploration. In a clear demonstration of the potential of post-processing to permit new applications, Fig. 11 shows the non-linear absorption measurements of a poly-crystalline core optical fibre that was tapered from $12 \mu \mathrm{m}$ to $2 \mu \mathrm{m}$ using the hot-filament method [33]. This result represents the first report of non-linear optical processes in poly-silicon waveguides in any format.

\subsection{Optical-electrical conversion}

There have been a number of successful attempts to produce optical detectors in the silicon optical fibre format $[54,55]$, however, the wavelengths that can be detected are limited to those of traditional silicon detectors $(0.3 \mu \mathrm{m}$ to $1.1 \mu \mathrm{m})$, thus precluding their use for telecoms applications. Post-processing techniques, in particular laser processing, present the intriguing possibility of extending this detection range. For example, the strained silicon described in Ref. [11] has been shown to detect light at wavelengths exceeding $2 \mu \mathrm{m}$, suggesting that laser annealed silicon could be used for detecting telecoms wavelengths. Furthermore, one could consider using the local increase in intensity produced in the waist of a tapered fibre as a means of producing a two-photon absorption facilitated detector. In addition, the fibres are being explored as a source material for the fabrication of in-fibre detector arrays and extracted semiconductor particles for p-n hybrid devices $[43,44]$.

Silicon optical fibres have also been used as solar cell precursor materials. Long lengths (metre-scale) of solar thread have been produced via the HPCVD method [56] and fibres produced by the MCD process have been 'cut and stacked' to produce a planar 
array $[57,58]$. The later experiments undertaken by Martinsen et al showed that they could substantially increase the purity of the silicon from solar cell grade to electronics grade using the drawing process in an analogue of the zone refining process, and it is envisaged that the tapering and laser processing could reduce impurities further. As the crystalline, optical and electronic properties of these materials continue to improve, there is a bright future for their development as fibre, fabric and array-based optoelectronic elements.

\subsection{Terahertz waveguides}

Silicon has been predicted to be a useful material for guiding terahertz radiation as it has a constant refractive index and relatively low absorption over this frequency range [59]. Thus this opens the door for these fibres to find use in this increasingly important frequency regime, where potential applications include security, medical imaging, quality control, environmental monitoring, to name but a few [60-62]. Compared to their planar counterparts, silicon fibres offer a number of useful advantages such as extended waveguiding lengths, flexibility and polarization independence, if required. In order to ensure that the radiation is well guided in the semiconductor core, away from the silica cladding, silicon diameters of several hundred's of micrometres would be required, making the molten core drawing technique the optimum fabrication approach. However, by considering different cladding material options and/or silicon-metal heterostructures, smaller and more flexible fibres could be designed. Post-processing of these fibres will provide an extra degree of freedom in device design, for example, index modification and tapering could be used to alter the waveguiding characteristics to produce gratings and mode-transformers.

\section{Conclusion}

The post-processing of semiconductor optical fibres is a rapidly growing field and though this review is focussed on silicon-based fibres, there is an ever-expanding catalogue of core types, such as germanium [45,63], zinc selenide [64], selenium telluride [65] and indium antimonide [66]. It is anticipated that the techniques that have been outlined will

be directly transferable to these materials facilitating a fruitful future for this research field.

It is incredibly encouraging that post-processing of semiconductor optical fibres has seen losses reduce dramatically and that they are now commensurate with silicon photonics waveguides. However, it is important to note that low-loss fibres at singlemode core dimensions have not yet been realised [67], though the hot-filament tapering method is now producing fibres that are approaching this critical milestone. The hot-filament method can also be used to produce silicon fibres with tapered profiles that are mode-matched and impedance-matched with standard optical fibres, thus 
greatly improving integration [29]. This, the added resolution, and the optoelectronic functionality provided by laser processing suggest that the silicon fibre is close to becoming a viable technology for next generation photonics networks.

\section{Acknowledgments}

The authors acknowledge their colleagues and students who have provided valuable contributions to the cited work, particularly L. Shen, F. Suhailin, L. Xiao, M. Fokine, S. Song, Y. Franz, J. Sparks, T. Day, J. Badding, and J. Ballato. Financial support has been provided by EPSRC. The data presented in this article is available from the corresponding author on request.

\section{References}

[1] B. Jalali and S. Fathpour. Silicon photonics. J. Lightwave Technol., 24:4600, 2006.

[2] R. H. Stolen and A. Ashkin. Optical Kerr effect in glass waveguide. Appl. Phys. Lett., 22:294, 1973.

[3] P. Mehta, N. Healy, N. F. Baril, P. J. A. Sazio, J. V. Badding, and A. C. Peacock. Nonlinear transmission properties of hydrogenated amorphous silicon core optical fibers. Opt. Express, 18:16826, 2010.

[4] L. Shen, N. Healy, L. Xu, H. Y. Cheng, T. D. Day, J. H. V. Price, J. V. Badding, and A. C. Peacock. Four-wave mixing and octave-spanning supercontinuum generation in a small core hydrogenated amorphous silicon fiber pumped in the mid-infrared. Opt. Lett., 39:5721, 2014.

[5] P. Mehta, N. Healy, J. R. Sparks, T. D. Day, P. J. A. Sazio, J. V. Badding, and A. C. Peacock. All-optical modulation using two-photon absorption in silicon core optical fibers. Opt. Express, 19:19078, 2011.

[6] A. C. Peacock, J. R. Sparks, and N. Healy. Semiconductor optical fibres: progress and opportunities. Laser Photon. Rev., 8:53, 2014.

[7] Gordon E Moore. Cramming more components onto integrated circuits. Proceedings of the IEEE, 86(1):82-85, 1998.

[8] Y. Sun, S. E. Thompson, and T. Nishida. Physics of strain effects in semiconductors and metaloxide-semiconductor field-effect transistors. Journal of Applied Physics, 101(10):104503, 2007.

[9] A. B. Sproul and M. A. Green. Improved value for the silicon intrinsic carrier concentration from 275 to 375 k. Journal of Applied Physics, 70(2):846-854, 1991.

[10] S.W. Bedell, A. Khakifirooz, and D.K. Sadana. Strain scaling for cmos. MRS Bulletin, 39(2):131137, 2014.

[11] N. Healy, S. Mailis, N. M. Bulgakova, P. J. A. Sazio, T. D. Day, J. R. Sparks, H. Y. Cheng, J. V. Badding, and A. C. Peacock. Extreme electronic bandgap modification in laser-crystallized silicon optical fibres. Nat. Mater., 13:1122, 2014.

[12] P. J. A. Sazio, A. Amezcua-Correa, C. E. Finlayson, J. R. Hayes, T. J. Scheidemantel, N. F. Baril, B. R. Jackson, D.-J. Won, F. Zhang, E. R. Margine, V. Gopalan, V. H. Crespi, and J. V. Badding. Microstructured optical fibers as high-pressure microfluidic reactors. Science, 311:1583, 2006.

[13] J. Ballato, T. Hawkins, P. Foy, B. Yazgan-Kokuoz, C. McMillen, L. Burka, S. Morris, R. Stolen, and R. Rice. Advancements in semiconductor core optical fiber. Opt. Fiber Technol., 16:399, 2010.

[14] P. J. Roberts, F. Couny, H. Sabert, B. J. Mangan, D. P. Williams, L. Farr, M. W. Mason, A. Tomlinson, T. A. Birks, J. C. Knight, and P. St. J. Russell. Ultimate low loss of hollow-core photonic crystal fibres. Opt. Express, 13:236, 2005. 
[15] N. Healy, L. Lagonigro, J. R. Sparks, S. Boden, P. J. A. Sazio, J. V. Badding, and A. C. Peacock. Polycrystalline silicon optical fibers with atomically smooth surfaces. Opt. Lett., 36:2480, 2011.

[16] N. F. Baril, R. He, T. D. Day, J. R. Sparks, B. Keshavarzi, M. Krishnamurthi, A. Borhan, V. Gopalan, A. C. Peacock, N. Healy, P. J. A. Sazio, and J. V. Badding. Low loss silicon fibers for photonic applications. J. Am. Chem. Soc., 134:19, 2011.

[17] L. Lagonigro, N. Healy, J. R. Sparks, N. F. Baril, P. J. A. Sazio, J. V. Badding, and A. C. Peacock. Low loss silicon fibers for photonic applications. Appl. Phys. Lett., 96:041105, 2010.

[18] Rongrui He, Todd D. Day, Justin R. Sparks, Nichole F. Sullivan, and John V. Badding. High pressure chemical vapor deposition of hydrogenated amorphous silicon films and solar cells. Advanced Materials, 28(28):5939-5942, 2016.

[19] J. Ballato, T. Hawkins, P. Foy, R. Stolen, B. Kokuoz, M. Ellison, C. McMillen, J. Reppert, A. M. Rao, M. Daw, S. Sharma, R. Shori, O. Stafsudd, R. R. Rice, and D. R. Powers. Silicon optical fiber. Opt. Express, 16:18675, 2008.

[20] B. Scott, W. Ke, and G. Pickrell. Fabrication of n-type silicon optical fibers. Photon. Technol. Lett., 21:1798, 2009.

[21] E. Nordstrand, A. Dibbs, A. Eraker, and U. J. Gibson. Alkaline oxide interface modifiers for silicon fiber production. Opt. Mater. Express, 3:651, 2013.

[22] C. Hou, X. Jia, L. Wei, S.-C. Tan, X. Zhao, J. Joannopoulos, and Y. Fink. Crystalline silicon core fibres from aluminium core preforms. Nat. Comm., 6:6248, 2015.

[23] C. Lin, J. Chen, and L. Wang. High-Q Si microsphere resonators fabricated from Si-cored fibers for WGMs excitation. IEEE Photon Technol. Lett., 27:1355, 2015.

[24] AC Peacock, UJ Gibson, and John Ballato. Silicon optical fibres-past, present, and future. Advances in Physics: X, 1(1):114-127, 2016.

[25] J. Ballato, T. Hawkins, P. Foy, S. Morris, N. K. Hon, B. Jalali, and R. Rice. Silica-clad crystalline germanium core optical fibers. Opt. Lett., 36:687, 2011.

[26] B. L. Scott and G. R. Pickrell. Silicon optical fiber diameter dependent grain size. J. J. Cryst. Growth, 371:134, 2013.

[27] Michael Fokine, Antreas Theodosiou, Seunghan Song, Thomas Hawkins, John Ballato, Kyriacos Kalli, and Ursula J. Gibson. Laser structuring, stress modification and bragg grating inscription in silicon-core glass fibers. Opt. Mater. Express, 7(5):1589-1597, May 2017.

[28] S. Morris, T. Hawkins, P. Foy, C. McMillen, J. Fan, L. Zhu, R. Stolen, R. Rice, and J. Ballato. Reactive molten core fabrication of silicon optical fiber. Opt. Mater. Express, 1:1141, 2011.

[29] Haonan Ren, Ozan Aktas, Yohann Franz, Antoine F. J. Runge, Thomas Hawkins, John Ballato, Ursula J. Gibson, and Anna C. Peacock. Tapered silicon core fibers with nano-spikes for optical coupling via spliced silica fibers. Opt. Express, 25(20):24157-24163, Oct 2017.

[30] T. A. Birks and Y. W. Li. The shape of fiber tapers. J. Lightwave Technol., 10:432, 1992.

[31] N. Healy, J. R. Sparks, P. J. A. Sazio, J. V. Badding, and A. C. Peacock. Tapered silicon optical fibers. Opt. Express, 18:7596, 2010.

[32] C. McMillen, G. Brambilla, S. Morris, T. Hawkins, P. Foy, N. Broderick, E. Koukharenko, R. Rice, and J. Ballato. On crystallographic orientation in crystal core optical fibers ii: Effects of tapering. Opt. Mater., 35:93, 2012.

[33] F. H. Suhailin, L. Shen, N. Healy, L. Xiao, M. Jones, T. Hawkins, J. Ballato, U. J. Gibson, and A. C. Peacock. Tapered polysilicon core fibers for nonlinear photonics. Opt. Lett., 41:1360, 2016.

[34] N. Healy, M. Fokine, Y. Franz, T. Hawkins, M. Jones, J. Ballato, A. C. Peacock, and U. J. Gibson. $\mathrm{CO}_{2}$ laser-induced directional recrystallization to produce single crystal silicon-core optical fibers with low loss. Adv. Optical Mater., DOI: 10.1002/adom.201500784, 2016.

[35] Y. Franz, A. F. J. Runge, H. Ren, N. Healy, K. Ignatyev, M. Jones, T. Hawkins, J. Ballato, U. J. Gibson, and A. C. Peacock. Material properties of tapered crystalline silicon core fibers. Opt. Mater. Express, 7:2055, 2017.

[36] Xiaoyu Ji, Shiming Lei, Shih-Ying Yu, Hiu Yan Cheng, Wenjun Liu, Nicolas Poilvert, Yihuang 
Xiong, Ismaila Dabo, Suzanne E. Mohney, John V. Badding, and Venkatraman Gopalan. Singlecrystal silicon optical fiber by direct laser crystallization. ACS Photonics, 4(1):85-92, 2017.

[37] N. Healy, S. Mailis, T. D. Day, P. J. A. Sazio, J. V. Badding, and A. C. Peacock. Laser crystallisation of semiconductor core optical fibres. In 2013 Conference on Lasers Electro-Optics Europe International Quantum Electronics Conference CLEO EUROPE/IQEC, pages 1-1, May 2013.

[38] Xiaoyu Ji, Shih-Ying Yu, Shiming Lei, Hiu Yan Cheng, Subhasis Chaudhuri, Wenjun Liu, Suzanne Mohney, John Badding, and Venkatraman Gopalan. Single crystal small core semiconductor optical fibers for all-fiber optoelectronics. In Conference on Lasers and Electro-Optics, page SM2K.5. Optical Society of America, 2017.

[39] David A. Coucheron, Michael Fokine, Nilesh Patil, Dag Werner Breiby, Ole Tore Buset, Noel Healy, Anna C. Peacock, Thomas Hawkins, Max Jones, John Ballato, and Ursula J. Gibson. Laser recrystallization and inscription of compositional microstructures in crystalline sige-core fibres. 7:13265 EP -, Oct 2016. Article.

[40] Yohann Franz, Antoine F. Runge, Swe Z. Oo, Noel Healy, Gregorio Martinez-Jimenez, Ali Z. Khokhar, Antulio Tarazona, Harold M. Chong, Sakellaris Mailis, and Anna C. Peacock. Laser annealing of low temperature deposited silicon waveguides. In Conference on Lasers and ElectroOptics, page SM3K.4. Optical Society of America, 2017.

[41] Yoon Ho Daniel Lee and Michal Lipson. Back-end deposited silicon photonics for monolithic integration on cmos. IEEE Journal of Selected Topics in Quantum Electronics, 19(2):82002078200207, 2013.

[42] N. Gupta, C. McMillen, R. Singh, R. Podila, A. M. Rao, T. Hawkins, P. Foy, S. Morris, R. Rice, K. F. Poole, L. Zhu, and J. Ballato. Annealing of silicon optical fibers. J. Appl. Phys., 110:093107, 2011.

[43] Alexander Gumennik, Etgar C. Levy, Benjamin Grena, Chong Hou, Michael Rein, Ayman F. Abouraddy, John D. Joannopoulos, and Yoel Fink. Confined in-fiber solidification and structural control of silicon and silicon-germanium microparticles. 114(28):7240-7245, 2017.

[44] Alexander Gumennik, Lei Wei, Guillaume Lestoquoy, Alexander M Stolyarov, Xiaoting Jia, Paul H Rekemeyer, Matthew J Smith, Xiangdong Liang, Benjamin Jb Grena, Steven G Johnson, et al. Silicon-in-silica spheres via axial thermal gradient in-fibre capillary instabilities. Nature communications, 4:2216, 2013.

[45] J Ballato, T Hawkins, P Foy, B Yazgan-Kokuoz, R Stolen, C McMillen, NK Hon, B Jalali, and R Rice. Glass-clad single-crystal germanium optical fiber. Optics express, 17(10):8029-8035, 2009.

[46] Ziwen Zhao, Xueli Cheng, Ting He, Fei Xue, Wei Zhang, Na Chen, Jianxiang Wen, Xianglong Zeng, and Tingyun Wang. Effect of controlling recrystallization from the melt on the residual stress and structural properties of the silica-clad ge core fiber. Optical Fiber Technology, 37:6-10, 2017.

[47] Ziwen Zhao, Xueli Cheng, Fei Xue, Ting He, and Tingyun Wang. Effect of annealing temperature on the stress and structural properties of germanium core fibre. Journal of Crystal Growth, 2017.

[48] S. Chaudhuri, J. R. Sparks, X. Ji, M. Krishnamurthi, L. Shen, N. Healy, A. C. Peacock, V. Gopalan, and J. V. Badding. Crystalline silicon optical fibers with low optical loss. ACS Photonics, 3:378384, 2016.

[49] Hicham El Hamzaoui, Géraud Bouwmans, Bruno Capoen, Na Chen, Marc Douay, and Mohamed Bouazaoui. Sol-gel silica glass-cladding semiconductor-core optical fiber. Materials Today Communications, 11:179-183, 2017.

[50] P. Mehta, N. Healy, T. D. Day, J. V. Badding, and A. C. Peacock. Ultrafast wavelength conversion via cross-phase modulation in hydrogenated amorphous silicon optical fibers. Opt. Express, 20:26110, 2012.

[51] A. C. Turner, C. Manolatou, B. S. Schmidt, M. Lipson, M. A. Foster, J. E. Sharping, and 
A. L. Gaeta. Tailored anomalous group-velocity dispersion in silicon channel waveguides. Opt. Express, 14:4357, 2006.

[52] A. C. Peacock. Soliton propagation in tapered silicon core fibers. Opt. Lett., 35:3697, 2010.

[53] Anna Peacock and Noel Healy. Parabolic pulse generation in tapered silicon fibers. Opt. Lett., 35(11):1780-1782, Jun 2010.

[54] R. He, P. J. A. Sazio, A. C. Peacock, N. Healy, J. R. Sparks, M. Krishnamurthy, V. Gopalan, and J. V Badding. Integration of gigahertz-bandwidth semiconductor devices inside microstructured optical fibres. Nat. Photon., 6:174, 2012.

[55] D Homa, A Cito, G Pickrell, C Hill, and B Scott. Silicon fiber with pn junction. Applied Physics Letters, 105(12):122110, 2014.

[56] Rongrui He, Todd D Day, Mahesh Krishnamurthi, Justin R Sparks, Pier JA Sazio, Venkatraman Gopalan, and John V Badding. Silicon p-i-n junction fibers. Advanced Materials, 25(10):14611467, 2013.

[57] Fredrik A Martinsen, Benjamin K Smeltzer, John Ballato, Thomas Hawkins, Max Jones, and Ursula J Gibson. Light trapping in horizontally aligned silicon microwire solar cells. Optics express, 23(24):A1463-A1471, 2015.

[58] Fredrik A Martinsen, BK Smeltzer, M Nord, T Hawkins, John Ballato, and UJ Gibson. Siliconcore glass fibres as microwire radial-junction solar cells. Scientific reports, 4, 2014.

[59] D. Grischkowsky, S. Keiding, M. van Exter, and Ch. Fattinger. Far-infrared time-domain spectroscopy with terahertz beams of dielectrics and semiconductors. J. Opt. Soc. Am. B, 7:2006, 1990.

[60] M. Tonouchi. Cutting-edge terahertz technology. Nat. Photonics, 1:97, 2007.

[61] Ruth M Woodward, Bryan E Cole, Vincent P Wallace, Richard J Pye, Donald D Arnone, Edmund H Linfield, and Michael Pepper. Terahertz pulse imaging in reflection geometry of human skin cancer and skin tissue. Physics in Medicine \& Biology, 47(21):3853, 2002.

[62] Daniel Clery. Terahertz on a chip. Science, 297(5582):763-763, 2002.

[63] P. Mehta, M. Krishnamurthi, N. Healy, N. F. Baril, J. R. Sparks, P. J. A. Sazio, V. Gopalan, J. V. Badding, and A. C. Peacock. Mid-infrared transmission properties of amorphous germanium optical fibers. Appl. Phys. Lett., 97:071117, 2010.

[64] J. Sparks, R. He, N. Healy, M. Krishnamurthi, A. C. Peacock, P. J. A. Sazio, V. Gopalan, and J. V. Badding. Zinc selenide optical fibers. Adv. Mater., 23:1647, 2011.

[65] G. Tang, Q. Qian, X. Wen, X. Chen, W. Liu, M. Sun, and Z. Yang. Reactive molten core fabrication of glass-clad $\mathrm{Se}_{0.8} \mathrm{Te}_{0.2}$ semiconductor core optical fibers. Opt. Express, 23:23624-23633, 2015.

[66] J. Ballato, T. Hawkins, P. Foy, C. McMillen, L. Burka, J. Reppert, R. Podila, A. M. Rao, and R. R. Rice. Binary III-V semiconductor core optical fiber. Opt. Express, 18:4972, 2010.

[67] A. C. Peacock, P. Mehta, P. Horak, and N. Healy. Nonlinear pulse dynamics in multimode silicon core optical fibers. Opt. Lett., 37:3351, 2012. 